\title{
Comparative genomics reveals phylogenetic distribution patterns of secondary metabolites in Amycolatopsis species
}

Martina Adamek ${ }^{1,2}$, Mohammad Alanjary ${ }^{1,2}$, Helena Sales-Ortells ${ }^{1,2}$, Michael Goodfellow ${ }^{3}$, Alan T. Bull4, Anika Winkler ${ }^{5}$, Daniel Wibberg ${ }^{5}$, Jörn Kalinowski ${ }^{5}$ and Nadine Ziemert ${ }^{1,2^{*}}$ (D)

\begin{abstract}
Background: Genome mining tools have enabled us to predict biosynthetic gene clusters that might encode compounds with valuable functions for industrial and medical applications. With the continuously increasing number of genomes sequenced, we are confronted with an overwhelming number of predicted clusters. In order to guide the effective prioritization of biosynthetic gene clusters towards finding the most promising compounds, knowledge about diversity, phylogenetic relationships and distribution patterns of biosynthetic gene clusters is necessary.

Results: Here, we provide a comprehensive analysis of the model actinobacterial genus Amycolatopsis and its potential for the production of secondary metabolites. A phylogenetic characterization, together with a pan-genome analysis showed that within this highly diverse genus, four major lineages could be distinguished which differed in their potential to produce secondary metabolites. Furthermore, we were able to distinguish gene cluster families whose distribution correlated with phylogeny, indicating that vertical gene transfer plays a major role in the evolution of secondary metabolite gene clusters. Still, the vast majority of the diverse biosynthetic gene clusters were derived from clusters unique to the genus, and also unique in comparison to a database of known compounds. Our study on the locations of biosynthetic gene clusters in the genomes of Amycolatopsis' strains showed that clusters acquired by horizontal gene transfer tend to be incorporated into non-conserved regions of the genome thereby allowing us to distinguish core and hypervariable regions in Amycolatopsis genomes.

Conclusions: Using a comparative genomics approach, it was possible to determine the potential of the genus Amycolatopsis to produce a huge diversity of secondary metabolites. Furthermore, the analysis demonstrates that horizontal and vertical gene transfer play an important role in the acquisition and maintenance of valuable secondary metabolites. Our results cast light on the interconnections between secondary metabolite gene clusters and provide a way to prioritize biosynthetic pathways in the search and discovery of novel compounds.
\end{abstract}

Keywords: Amycolatopsis, Genome mining, Comparative genomics, Biosynthetic gene cluster, Gene cluster family, Secondary metabolite diversity, Phylogeny, Natural products, Evolution

\footnotetext{
* Correspondence: nadine.ziemert@uni-tuebingen.de

${ }^{1}$ Interfaculty Institute of Microbiology and Infection Medicine Tübingen,

Microbiology/Biotechnology, University of Tübingen, Tübingen, Germany

${ }^{2}$ German Centre for Infection Research (DZIF), Partner Site Tübingen,

Tübingen, Germany

Full list of author information is available at the end of the article
}

(c) The Author(s). 2018 Open Access This article is distributed under the terms of the Creative Commons Attribution 4.0 International License (http://creativecommons.org/licenses/by/4.0/), which permits unrestricted use, distribution, and reproduction in any medium, provided you give appropriate credit to the original author(s) and the source, provide a link to the Creative Commons license, and indicate if changes were made. The Creative Commons Public Domain Dedication waiver (http://creativecommons.org/publicdomain/zero/1.0/) applies to the data made available in this article, unless otherwise stated. 


\section{Background}

The value of bacterial secondary metabolites for medical applications, as pharmaceuticals, especially anti-infectives, but also for industrial use is indisputable $[1,2]$. Furthermore, the demand for the discovery of novel compounds for medical applications is urgent, especially in the light of the increasing antibiotic resistance to drugs currently in use [3]. To facilitate the discovery of novel compounds, bacterial genome sequences are screened for genome regions that are likely to code for the production of secondary metabolites. This bioinformatics approach is the first important step in the genome mining pipeline that is necessary to guide the discovery of novel compounds $[4,5]$. The secondary metabolite machinery of bacteria is mainly organized into several diverse clusters, called biosynthetic gene clusters (BGCs), which contain biosynthesis genes in close physical proximity. BGCs encoding for closely related biosynthetic pathways that produce highly similar chemical compounds are summarized under the term gene cluster families (GCFs). Polyketide synthase (PKS) and non-ribosomal peptide synthetase (NRPS) gene clusters are huge megasynthases that produce natural products by a multimodular assembly line in a series of chemical condensation reactions [6]. Other notable classes include ribosomally synthesized and post-translationally modified peptides (RiPPs) and terpenes [7, 8].

Recent comparative genomics approaches have shown that the potential for bacteria to produce secondary metabolites is much more promising than previously thought, as many actinobacterial genomes harbor 20-29 BGCs on average [9]. With the currently available tools, detection of putative BGCs is fast and simple [10]. It is now feasible to detect thousands of putative BGCs. To guide the discovery of the most promising novel compounds, it is important to understand the distribution patterns of BGCs. Therefore, knowledge about the diversity, environmental distribution and phylogenetic relationships of BGCs in the context of their environmental function is paramount.

In contrast to primary metabolites, bacterial secondary metabolites are not necessary for the immediate survival of the bacterium, but are important for adaption, as well as for fitness advantages in specific natural habitats. Early hypotheses suggested that bacteria mainly produce secondary metabolites with antibiotic activity for defense purposes, more recent studies show that these secondary metabolites also play a key role as signaling molecules $[11,12]$. Furthermore, they have been shown to be involved in complex mutualistic relationships in their specific environment [13]. Yet, the complex functions of secondary metabolites in their natural environment remain poorly understood.

Previous approaches to characterize secondary metabolite gene clusters used different methods to sort BGCs into related GCFs [14-16]. It was shown that on one hand BGC distribution was correlated with species phylogeny while on the other hand the vast BGC diversity could not be explained by vertical evolution. Furthermore, distinct taxa, or even distinct species, show remarkable differences in their BGCs. This leaves open questions concerning the main mechanisms for secondary metabolite evolution. Because of these taxonomic differences, it is necessary to characterize many different bacterial genera in order to evaluate the diversity of BGCs and the mechanisms leading to their diversification. This knowledge should help us to predict where to seek novel secondary metabolites, and to estimate if the search for novel producers should be based on phylogeny, geography or on specific microenvironments. Classifying GCFs enables us to further prioritize BGCs with respect to their novelty and to predict their structural scaffolds [4].

In this work, we focus on the actinomycete genus Amycolatopsis as a model system for an in-depth study of secondary metabolite gene clusters harbored by this genus. As of 2017, 69 different Amycolatopsis species have been validly named [17]. 41 genome sequences representing 28 different Amycolatopsis species are publicly available as complete or draft genome sequences. Amycolatopsis strains are ubiquitously distributed and have been isolated foremost from soil, but also from aquatic habitats, rock surfaces, and from clinical sources [18-23]. Only four Amycolatopsis species are known to have pathogenic properties [24, 25].

Amycolatopsis is already valued as a producer for the commercially used vancomycin and other glycopeptide antibiotics as well as for the production of the ansamycin rifamycin [26]. Other compounds with antibacterial, antifungal or antiviral properties that have been derived from Amycolatopsis strains are quartromycin [27], octacosamicin [28], chelocardin [29], kigamicin [30] and the macrotermycins A-D [31].

To explore the full potential of Amycolatopsis strains for the synthesis of secondary metabolites, we performed a comprehensive analysis of the secondary metabolite gene clusters in Amycolatopsis. We were able to elucidate the phylogenetic patterns in which biosynthetic gene clusters evolve and to reveal the huge genetic potential of members of this taxon to produce novel secondary metabolites.

\section{Results}

In order to characterize and compare members of the genus Amycolatopsis and to establish their potential for biosynthesis of secondary metabolites we used 43 Amycolatopsis genome sequences for a comparative genomics approach. In total, 41 of the 43 strains were derived from public databases and two strains, Amycolatopsis sp. H5 and KNN 50.9b, were newly sequenced. This Whole Genome Shotgun project has been deposited at 
DDBJ/ENA/GenBank under the accession NMUL00000000 (H5) and NMUK00000000 (KNN50.9b). The version described in this paper is version NMUL01000000 for Amycolatopsis sp. H5 and version NMUK01000000 for Amycolatopsis sp. KNN50.9b. Basic data for the newly sequenced strains are given in the supplementary material (Additional file 2: Table S1).

\section{Characterization of the genus Amycolatopsis}

To assess relationships between the sequenced Amycolatopsis strains we performed a multi locus sequence analysis (MLSA). Based on the concatenation of 7 housekeeping genes $(a t p D, c l p B, g a p A, \operatorname{gyr} B, n u o D, p y r H, r p o B)$ a maximum likelihood phylogenetic tree was generated for all of the 43 Amycolatopsis strains (Fig. 1a); Nocardia farcinina IFM10152 and Streptomyces avermitilis MA-4680 were used as outgroups. We were able to distinguish four major phylogenetic lineages containing the majority of the Amycolatopsis stains, from here on referred to as A, B, C and D. Six strains, namely A. halophila YIM 93223, A. marina
CGMCC 4.3568, A. nigrescens DSM 44992, A. sacchari DSM 44468, A. taiwanensis DSM 45107, and A. xylanica CPCC 202699 formed distinct single membered clades. It was not possible to detect any significant relationships between the phylogeny of Amycolatopsis strains and their origin (Additional file 3: Table S2). Members from the same phylogenetic clade were isolated from various geographic regions across the world. The majority of strains were isolated from diverse soils; the marine isolate A. marina CGMCC 4.3568 and the salt-lake isolate $A$. halophila YIM 93223 did not clade with any of the soil strains.

Discrepancies were observed in the assignment of strains delineated as Amycolatopsis orientalis. Among the strains in group $\mathrm{A}$ is the industrial vancomycin producer $A$. orientalis HCCB10007 which clades a significant distance away from the $A$. orientalis DSM 40040 T. Furthermore, $A$. orientalis DSM 46075 and DSM 43388 fell into clade $C$, even further away from the $A$. orientalis type strain. When comparing the MLSA tree with a $16 \mathrm{~S}$ rRNA tree based on sequences derived from genomic
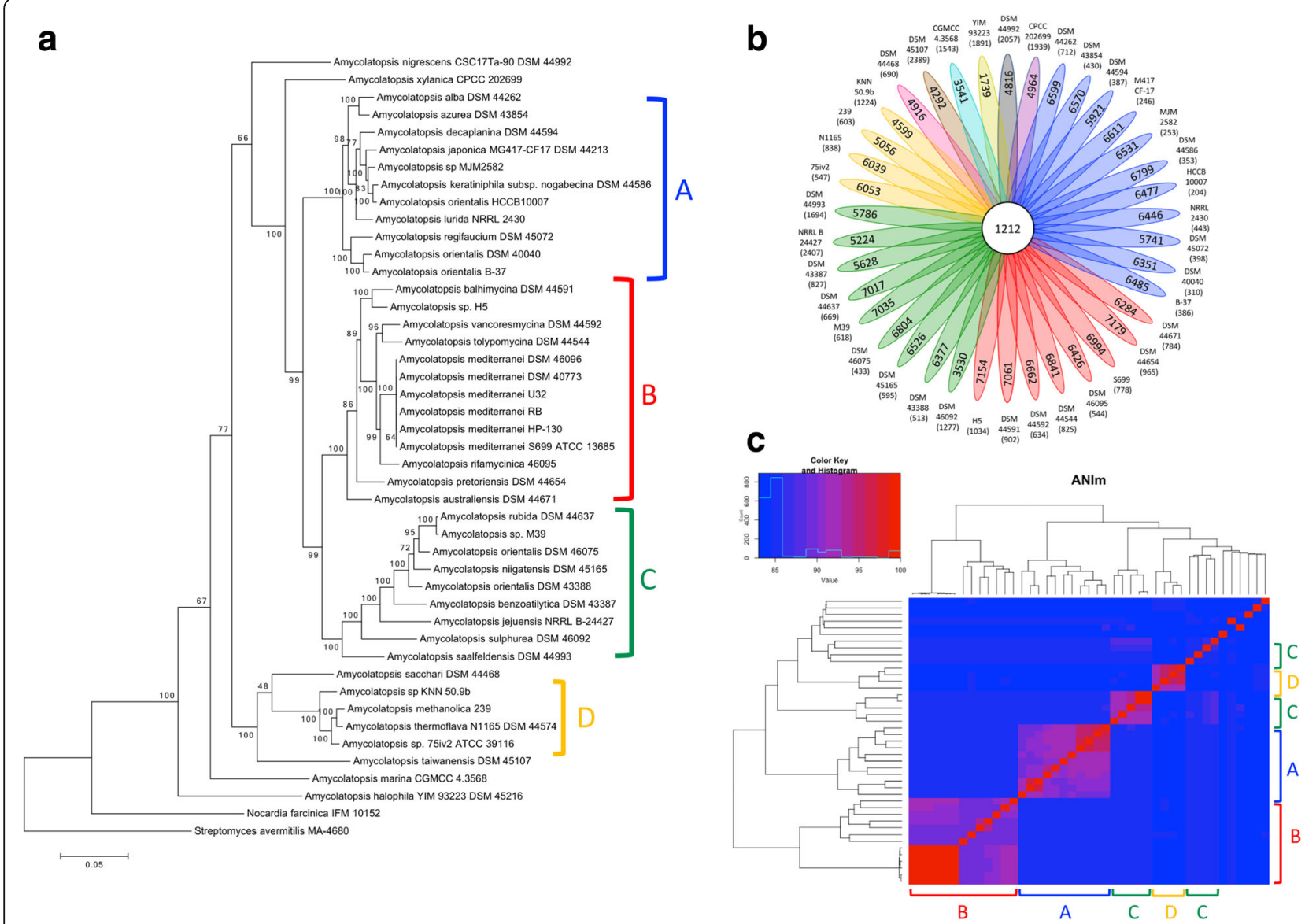

Fig. 1 Amycolatopsis phylogeny, core-/pan-genome and average nucleotide identity. a) Maximum likelihood tree based on a MLSA (concatenated sequences of $a t p D, c l p B, g a p A, g y r B, n u o D, p y r H$ and $r p o B$ ) of 43 members of the genus Amycolatopsis. Bootstrap values were calculated from 500 bootstrap repetitions. b) Flower diagram representing the core-, accessory- and pan-genome of the Amycolatopsis strains. c) Heatmap displaying relationships between Amycolatopsis strains based on ANIm values 
data (Additional file 1: Figure S1), similar discrepancies could be seen. A. orientalis HCCB10007 clades in close proximity to A. japonica DSM 44213, but not with the A. orientalis type strain DSM 40040. A. orientalis DSM 46075 and DSM 43388 clade with group $C$ strains as in the MLSA tree. However, in the 16S rRNA tree it could be clearly seen that the phylogenetic resolution is too low to distinguish Amycolatopsis strains on a species level. One problem here is that most Amycolatopsis strains have multiple, in some cases different, copies of the 16S rRNA gene. While the four clades (A-D) were basically the same in the $16 \mathrm{~S}$ rRNA tree as in the MLSA tree, in some cases the multiple 16S rRNA copies did not clade. This could be seen for example for $A$. orientalis $\mathrm{B}-37$ that clades among multiple copies of $A$. lurida $16 \mathrm{~S}$ rRNA genes, for $A$. decaplanina, which clusters with different copies of A. keratiniphila subsp. nogabecina, and for $A$. sacchari, which clades among $A$. sulphurea genes (Additional file 1: Figure S1).

In order to assess the genome similarity amongst the Amycolatopsis strains, a pan genome analysis was performed using the BPGA analysis tool [32]. To reduce any bias conferred by the 6 closely related and highly similar $A$. mediterranei genomes, only the $A$. mediterranei S699 genome was used as a reference for $A$. mediterranei. The pan-genome analysis revealed a core genome of 1212 genes with an accessory genome of 27,483 genes and 33,342 unique genes (Fig. 1b). The core-pan plot (Additional file 1: Figure S2) shows that the pan genome is likely to be extended if more genomes were added to the analysis, hence the pan genome is considered to be "open". The core genome curve levels off, therefore the addition of more genomes to the analysis will probably not change the core genome size significantly. The COG (Clusters of Orthologous Groups) analysis (Additional file 1: Figure S3) for core, accessory and unique genes revealed that the majority of the core genes are involved in translation and ribosomal structure biogenesis. Core, accessory and unique genes are all similarly involved in transcription and amino acid transport and metabolism. A remarkable number of unique and accessory genes are involved in the biosynthesis of secondary metabolites and in transport and catabolism. The majority of genes could only be linked to some general functions or to no function at all.

As group D strains and A. taiwanensis and A. halophila were clustering apart from the majority of the strains, we suspected they might represent novel taxa, distinct from the genus Amycolatopsis. Consequently, the average nucleotide identity based on MUMmer (ANIm) to distinguish strains at species level, and the percentage of conserved proteins (POCP) to distinguish strains at genus level, were calculated for all vs. all strains. The results, displayed as a heatmap (Fig. 1c), show that within the phylogenetic subgroups the strains have ANIm values of $89.8-96.8 \%$ (group A), 88.7-99.9\% (group B), 85.3-99.1\% (group C) and $84.4-96.5 \%$ (group D). For the strains that do not clade with any of the larger phylogenetic groups the ANIm values with the other strains ranged from $83.7-84.4 \%(A$. nigrescens), 83.5-85.0\% (A. xylanica), 83.6-86\% (A. marina) and $83.0-84.0 \%$ ( $A$. halophila). Comparing these values to the average ANI observed within other bacterial genera [33] shows that all Amycolatopsis stains are within average boundaries specified for a bacterial genus, hence their assignment to the genus Amycolatopsis is supported. Results of the POCP analysis (Additional file 4: Table S3) further confirm that except for A. halophila all of the Amycolatopsis strains have at least $50 \%$ conserved proteins, and therefore belong to the same genus, while $A$. halophila might be considered a different genus.

\section{Amycolatopsis biosynthetic gene clusters - diversity and phylogenetic affiliation}

To study the potential of the strains to produce secondary metabolites, all of the Amycolatopsis genomes were screened for candidate BGCs using the secondary metabolite identification pipeline antiSMASH. Because the estimation of precise cluster boundaries is a critical step when computationally comparing BGCs, all of the clusters detected with antiSMASH were manually curated [34]. A detailed overview on the distribution of BGCs with respect to their phylogenetic affiliation is given in Additional file 1: Figure S4.

In general, strains from the phylogenetic groups $\mathrm{A}$ and $B$ have a higher number of BGCs (A: on average 37 BGCs, range 34-45 BGCs; B: on average 34 BGCs, range 28-41 BGCs) than strains from group $C$ (on average 30 BGCs, range 22-38 BGCs). Within group D the lowest number of BGCs (on average 18 BGCs, range 14-20 BGCs) were identified. The genomes of $A$. sacchari and A. taiwanensis, which are distinctly related with group D, have 16 and 18 BGCs respectively. The strains from the isolated aqueous and saline environments harbor only 22 BGCs (A. marina) and 14 BGCs (A. halophila). In contrast, 43 and 41 BCGs were found in the genomes of the A. xylanica and A. nigrescens strains. When comparing the BGC representatives for the different phylogenetic clades, it can be seen that strains from groups A and B have remarkably high numbers of PKS and NRPS genes compared to the group $C$ and D strains. The number of RiPP, terpene and other BGCs is fairly constant over the different phylogenetic subgroups, though the genome of the A. halophila strain lacks terpene BGCs. Overall each strain added to the analysis contributed on average 6-7 new BGCs.

The relationship between the BGCs of each Amycolatopsis strain was assessed by manually sorting the identified BGCs to GCFs, according to cluster architecture and Blast similarity. A concise overview of the sorting rationale is given in Additional file 1: Figure S5. Overall 442 GCFs 
were distinguished, the majority of which were either PKS or NRPS. It is possible to distinguish between common GCFs (present in four or more strains), rare GCFs (present in 2-3 strains) and unique GCFs (present in only one strain).

The distribution of GCFs amongst the members of the genus Amycolatopsis is visualized in Fig. 2 as a presence/ absence map. It can be seen that Amycolatopsis strains with a high similarity in their BGC presence/absence patterns cluster together in the dendrogram. The patterns in the distribution of GCF in the main correlate with the species phylogeny. Comparing the BGCs and their phylogenetic affiliation, it can be seen that the common GCFs are usually present in all members of their phylogenetic clade and rarely cluster outside of their phylogenetic subgroups. The common PKS, NRPS and PKS/NRPS-hybrid clusters, as well as some of the RiPP families are mainly represented. Four terpene cluster families, one RiPP family and several clusters from the "others" category were present in the genomes of the majority of the Amycolatopsis strains. Additional file 1: Figure S6 shows the frequency of GCFs within the genus Amycolatopsis in detail. When comparing the distribution of GCFs, the conserved GCFs only account for a small proportion of the biosynthetic pathway diversity in Amycolatopsis, only 33\% are rare or common GCFs. A vast number of GCFs are represented by only a single member (67\% unique GCFs). The number of unique GCFs exceeds the common and occasional GCFs by a factor of two. These numbers emphasize the huge potential for strain specific diversification.
We also used a computational method to group BGCs into GCFs and visualized them by genetic networking. The resultant groups follow the similarity of their Pfam-domains in each cluster, as previously noted by Cimermancic et al. [16]. Using the Jaccard- and domain duplication index (DDI) as distance metrics a genetic network showing an all vs. all comparison of the Amycolatopsis BGCs was generated (Fig. 3a). The same color code as for the BGC-presence/absence map was used to distinguish between the BGC-classes. Most of the delineated GCFs corresponded to our previously defined GCFs. In Fig. 3a the BGCs that were previously linked to a specific secondary metabolite are highlighted. This encompasses the NRPS biosynthesis clusters encoding the albachelin and amychelin like siderophores, and the glycopeptide class of antibiotics. Furthermore, the polyketide clusters for rifamycin, ECO-0501, chelocardin and the macrotermycins are shown. The vast majority of strains harbored a 2-metyhlisoborneol encoding terpene BGC. All Amycolatopsis strains harbored the same ectoine BGC, which was excluded from further analyses because it should be considered as a primary metabolite. An example in which the automatically calculated GCFs differed from the manually sorted ones is shown in the Additional file 1: Figure S7.

To distinguish novel BGCs from known BGCs we used gene clusters deposited at the Minimum Information about a Biosynthetic Gene Cluster (MIBiG) database as a reference, which at the date of publication contained 1297 annotated BGCs of known compounds. A genetic network of all of the MIBiG BGCs together with all of

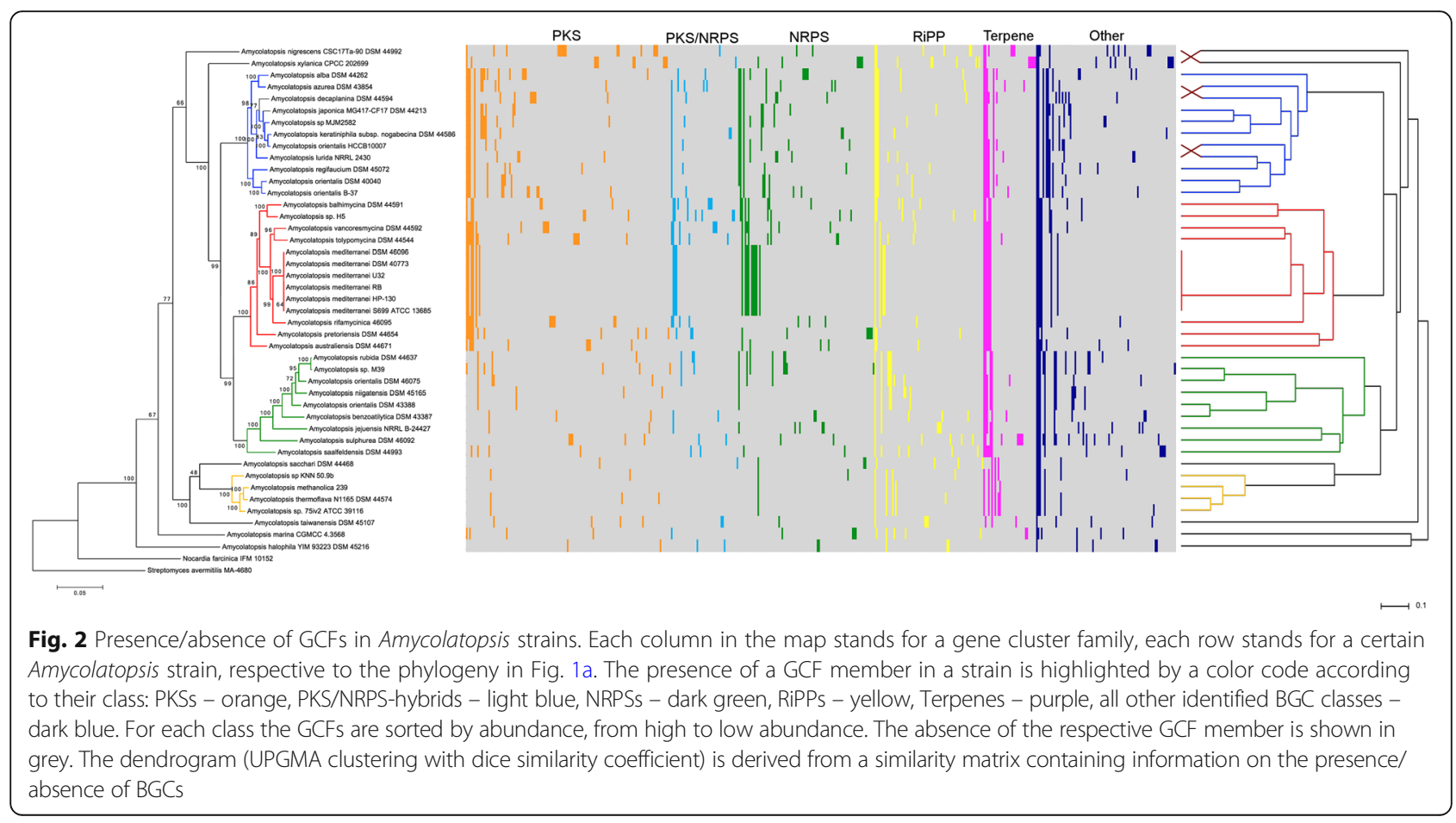




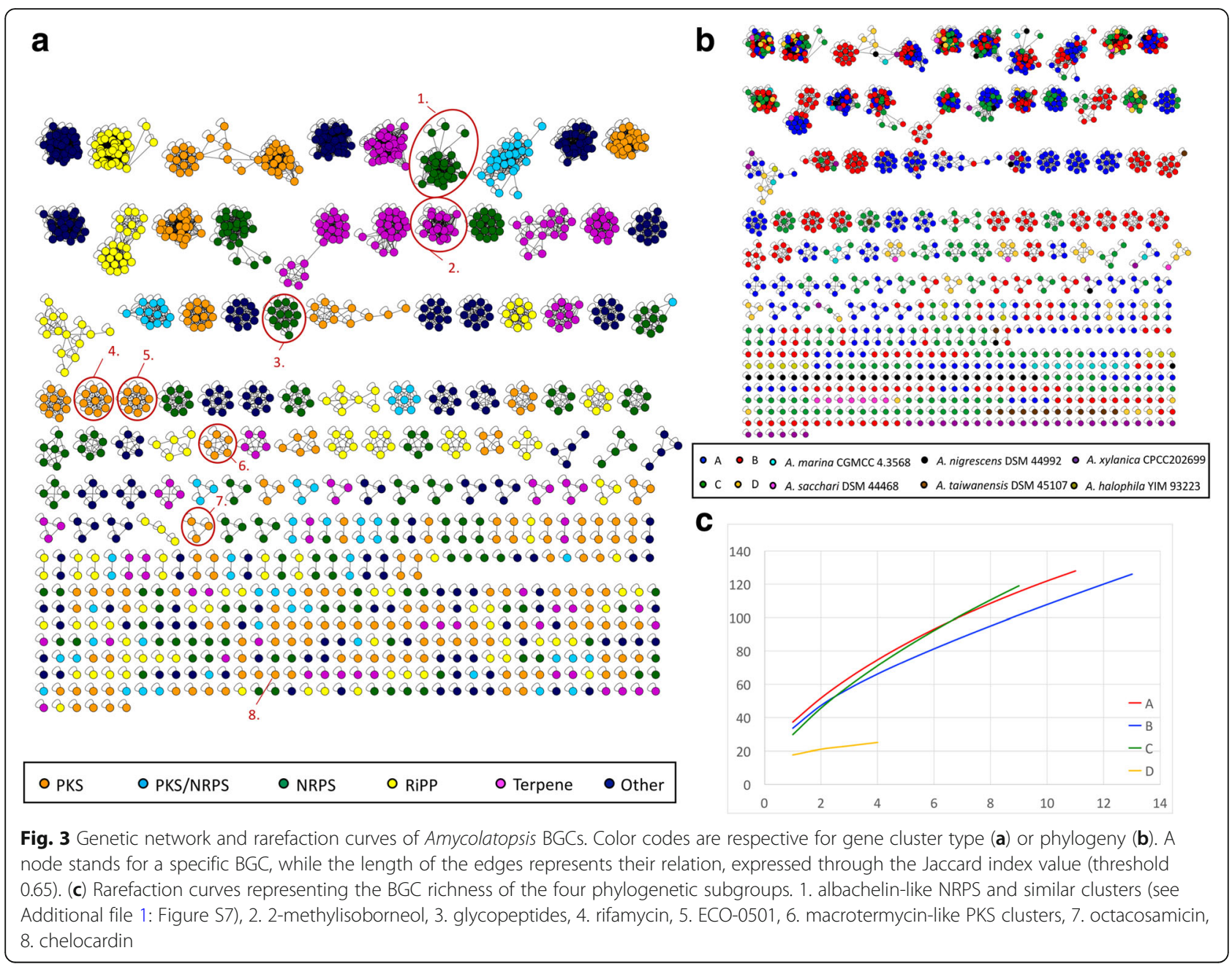

the Amycolatopsis BGCs was created, using the Cimermancic index (Additional file 1: Figure S8). It was possible to distinguish 1149 clusters, 388 of which were only found in the genomes of the Amycolatopsis strains, 742 were MIBiG only, and 19 consisted of Amycolatopsis and MIBiG clusters. Of the 388 Amycolatopsis only clusters 275 were singletons. These results provide further evidence of the huge diversity of Amycolatopsis BGCs and the immense potential this genus has for the detection of novel secondary metabolites.

To estimate further relationships between the Amycolatopsis phylogenetic groups and the GCFs we used a different color code for the nodes in the gene cluster network, according to the strains' phylogenetic affiliation (Fig. 3b). Of the 70 common GCFs network clusters 31 were specific for one phylogenetic group, 17 had members from two phylogenetic lineages, and 22 contained members of three or more different phylogenetic lineages. For the families with only two or three members, the numbers are too low to draw conclusions concerning the distribution of phylogenetic groups. The majority of the A. halophila, A. nigrescens, A. taiwanensis and A. xylanica BGCs remained singletons, while about half of the BGCs from A. marina clustered in several of the larger groups with mixed phylogeny. Some A. sacchari BGCs clustered with group D strains.

To assess BGC richness for a phylogenetic group a rarefaction curve, representing the abundance of BGCs per strain is shown (Fig. 3c); a steep slope of the curve indicates that it is likely that more novel BGCs will be discovered if more strains are sampled. A steep slope can be seen for all four phylogenetic groups, although that for group D is much lower. Therefore, we would expect that maximum diversity will be reached when sampling only a few more strains from group D. It can be concluded that new members of all of the phylogenetic groups have the potential to harbor yet undiscovered biosynthetic pathways. Plotting the relative number of BGCs per strain against the genome size (Additional file 1: Figure S9) revealed that phylogenetic clades A and B not only have the largest genomes but also harbor the highest number of BGCs. Members of clade C have comparably large genomes, but less BGCs while 
clade D strains have the smallest genomes and the lowest BGC numbers. Taken all together, the most promising phylogenetic groups for genome mining are represented by the clade A and B strains, as well as by the $A$. nigrescens and A. xylanica strains.

\section{BGC locations on the Amycolatopsis genomes}

The relative positions of the BGCs on the genomes can provide additional information about gene transfer, rearrangements and relationships of the BGCs. As all of the A. mediterranei strains showed the same BGCs in the same location, this species is only represented by $A$. mediterranei strain S699 in the subsequent analyses. Since only 11 out of the 38 Amycolatopsis genomes were in a complete state or available as draft genome with only one scaffold we assembled the draft genomes with multiple contigs as linearized pseudo contigs. For most of the complete genomes and the pseudo contigs synteny with the respective reference strain of their phylogenetic group is given. For the A. japonica and A. lurida genomes large scale rearrangements were observed that affected the position of the BGCs.

The position of each BGC was annotated on the complete genomes and pseudo contigs of all of the Amycolatopsis strains. Figure 4 shows the relative position of all common GCFs (with four or more members). Different patterns can be observed with respect to the distribution of BGCs throughout the Amycolatopsis genomes and pseudocontigs. Not only is the presence/absence of BGCs correlated with the phylogeny, but the location of most of the common BGCs is conserved within phylogenetic groups. This can be seen, for example, for "Lantipeptide BGC-1" and "Terpene BGC-6" which is always neighboring the "Other BGC-6" clusters (highlighted as grey squares in Fig. 4). For other GCFs the position on the genome is not fixed, examples are highlighted as grey circles in the Figure. This is seen best for PKS/NRPS BGC-4, which is distributed throughout phylogenetic clades $\mathrm{A}$ and $\mathrm{B}$ and is also present in the genome of $A$. marina. Another example of a BGC with a variable position is NRPS BGC-14, which is present in some members of phylogenetic clades A, B and C. Finally, an example of the huge diversity of BGCs, with respect to their locations on the genome and their phylogeny are the NRPS BGC-10 clusters, which are members of the glycopeptide family (highlighted with yellow stars in Fig. 4). All of the strains from the phylogenetic clade $A$ and two strains from group B harbor the glycopeptide BGC in different locations on the genome. For $A$. japonica and $A$. lurida it can be speculated that the different locations on their genome is due to genome rearrangements. The presence of the glycopeptide BGCs in the group B genomes of A. balhimycina and in the genomes of Amycolatopsis sp. $\mathrm{H} 5$ clearly indicates that these clusters have been acquired by horizontal gene transfer (HGT).
Taken together the common BGCs tend to be located in a broad central area on the genome, opposite to the replication origin oriC, located upstream form the $d n a A$ gene. These patterns can also be observed when all of the BGCs are taken into account. Additional file 1: Figure S10 shows the position of all of the BGCs on each of the linearized genomes and pseudocontigs.

Figure 5 shows the relative position for gene cluster types, such as terpenes, NRPS, and lantipeptides, on a circular genome model. This relative position is expressed as downstream distance (\%) from oriC. For the majority of cluster types the distribution is denser around a region opposite to the replication origin, while the regions flanking the replication origin tend to have less clusters. Exceptions from these patterns are represented by the lantipeptides, lassopeptides, aryl-polyenes and indoles, where about half of the clusters are located in a region near to the replication origin.

To finally compare BGC location with overall genome conservation within the phylogenetic groups, conserved regions and hypervariable regions were identified using a PARSNP core genome alignment. Because of the large genetic differences between the Amycolatopsis strains, it was not possible to detect genomic islands though core-regions and hypervariable regions were observed. It can be seen that the more closely related the strains, the smaller the hypervariable regions. It can be seen from Additional file 1: Figure S11 that for the majority of BGCs the location also corresponds with the hypervariable regions of the genome.

\section{Discussion}

Actinobacterial genome sequences have a much higher potential for the production of secondary metabolites than previously thought $[35,36]$. With recent advances in bioinformatic search algorithms, it is possible to identify novel biosynthesis pathways based on predictions drawn from bioinformatics, and thereby guide the discovery of novel compounds [4]. Nevertheless, little is known about the variety and the evolutionary interconnections between secondary metabolite gene clusters and species' phylogeny [37]. Doroghazi and Metcalf were able to portray the huge diversity of secondary metabolites in different actinomycete genera [38], but it is also apparent that the genomes of a single bacterial genus can harbor a wealth of undiscovered secondary metabolites [14, 39]. In order to study the diversity and relationships of secondary metabolites we focused on the genus Amycolatopsis, which is already known to produce valuable secondary metabolites [26], and to harbor a yet unknown potential for the discovery of new natural products.

To draw a comprehensive picture of the phylogenetic relations between the sequenced members of the genus Amycolatopsis a MLSA approach based on seven common housekeeping genes was used. At the 16S rRNA 


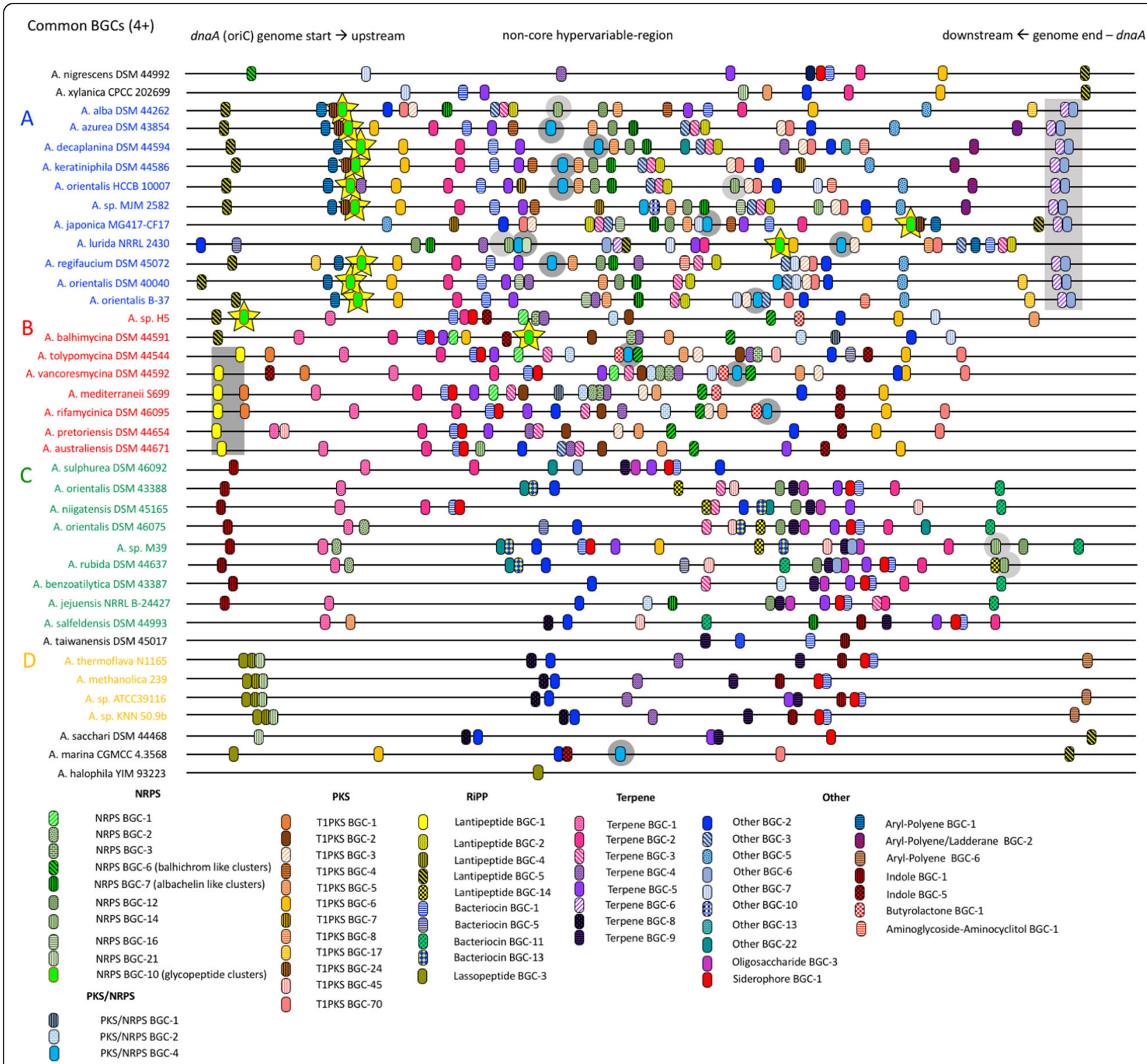

Fig. 4 The relative location of common BGCs on linearized genomes and pseudocontigs of Amycolatopsis. Examples for cluster families conserved in a phylogenetic group, which also share the same location are highlighted in gray squares. Examples for cluster families with a random distribution pattern are highlighted with gray circles. The glycopeptide as example for a cluster family with unusual distribution patterns are highlighted in yellow stars

level the similarity between strains is around $97 \%$ or higher [40], hence discrimination based only on $16 \mathrm{~S}$ rRNA data does not clearly identify relationships among members of the genus. In contrast, using MLSA, four major Amycolatopsis clades were detected. Furthermore, four isolates each formed a separate phylogenetic branch. By phylogenetic analysis based on 16S rRNA and an actinobacterial conserved gene, Tang et al. [41] delineated three types of Amycolatopsis stains: the mesophilic and moderately thermophilic $A$. orientalis clade (AOS), the mesophilic A. taiwanensis clade (ATS), and the thermophilic $A$. methanolica subclade (AMS). In our study we were able to further distinguish members of the AOS clade in there different phylogenetic subclades (clade A, B and C). The AMS is represented by Amycolatopsis group D, and the ATS clade only by $A$. taiwanensis. ANIm values underpinned these results, as ANI values within the subgroups were much higher than between them. ANI values below the $95 \%$ threshold are commonly used for species delineation [42]. On this basis, strains previously classified as A. orientalis HCCB10007, DSM 43388 and DSM 46075 were shown to be misclassified. No information regarding the original method of classification was available for $A$. orientalis DSM 43388 and DSM 46075. A. orientalis HCCB10007 was derived from the strain A. orientalis ATCC 43491 through physical and chemical mutageneses 

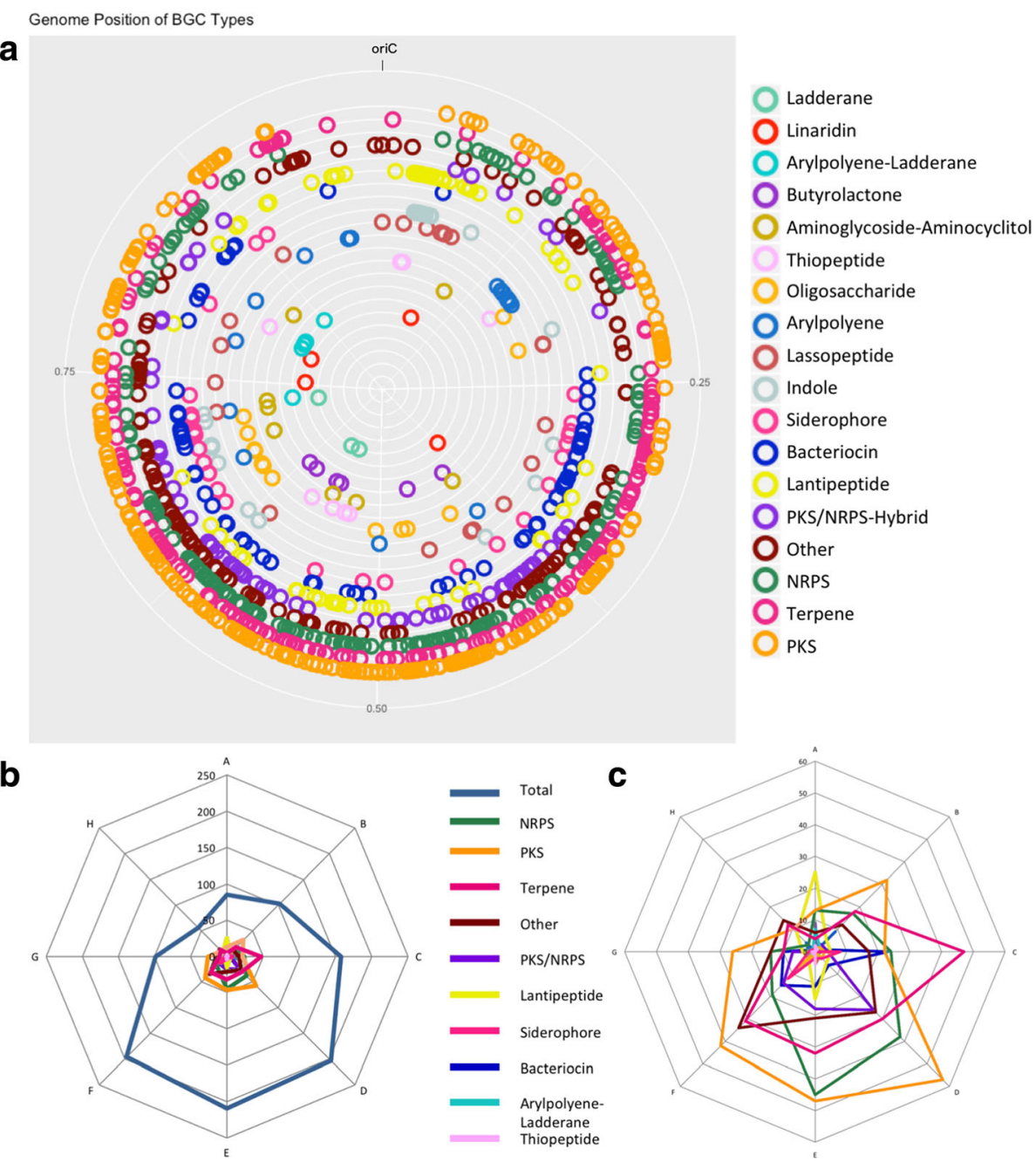

Fig. 5 Relative location and density of all BGCs on the circular Amycolatopsis genomes. a) Relative location of Amycolatopsis BGCs expressed as downstream distance (0.00-1.00) to the replication origin oriC $(=0.00)$. b) BGC density on certain areas of the circular Amycolatopsis genome (Total). c) BGC density on certain areas of the circular Amycolatopsis genome (main BGC classes)

[43]. This strain has originally been classified as Streptomyces orientalis, and has since been renamed twice (Nocardia orientalis and Amycolatopsis orientalis) [20, 44]. Consequently, we agree with the previous suggestion by Jeong et al. that stains DSM 46075 and DSM 43388 belong to novel Amycolatopsis species [45], while further studies are needed to establish if strain HCCB10007 belongs to the species $A$. keratiniphila.

Furthermore, POCP analysis showed that A. halophila, which was first classified based on $16 \mathrm{~S}$ rRNA sequencing [22], might represent a novel genus. In their study, evaluating the thresholds to define a novel genus based on the POCP values, Qin et al. suggested to consider the genome size for prokaryotic taxonomy [46]. A. halophila YIM 93223 also has a much smaller genome than other Amycolatopsis strains. Therefore, there is need to reevaluate the taxonomic status of this strain. Our results further emphasize the need to set new standards for the taxonomic classification of bacterial strains using genome sequences [47].

The majority of the Amycolatopsis strains were isolated from different soil types, but no correlation was found between their geographic distribution and phylogenetic relationships though the aquatic isolates, $A$. halophila and A. marina, did not cluster with the soil isolates. Tan et al. [48] investigated the phylogenetic diversity of different Amycolatopsis strains isolated from the same geographical and ecological habitat based on 16S rRNA sequencing. and showed that at the same site the strains fell into several phylogenetic groups which corresponded to the four phylogenetic subclades found in this study. Taken together these results suggest that there is no correlation between geography and phylogeny for Amycolatopsis soil isolates though phylogenetic diversity can be found in 
small, geographically close regions. The four Amycolatopsis sublineages are ubiquitously distributed and hence are not the consequence of adaption to a specific geographical region. In contrast, too little data are available to draw conclusions about the distribution of the aquatic isolates. Further, no correlation was found between the geographic distribution of strains and that of their BGCs, though a correlation was found between the species' phylogeny and the distribution of BGCs. Therefore, it can be concluded that taxonomy is a more important indicator of BGC distribution than geographic origin. This phenomenon has also been observed with the marine actinobacterium Salinispora [14]. In general, these data support the view that geographically distant but ecologically similar habitats share overlapping gene pools. [49]. The rarefaction curves for all of the phylogenetic groups (Fig. 3c) showed that sampling more Amycolatopsis genomes, will lead to the discovery of novel BGCs even if the sampling was restricted to the same geographic regions and soil types.

Core-/pan-genome analysis revealed that members of the genus Amycolatopsis shared a core genome of 1212 genes and a pan genome of 27,483 accessory and 33,342 unique genes. So far only few core-/pan-genome studies have been carried out for actinobacteria with comparably large genomes $(5-10 \mathrm{Mb})$. A study on 17 Streptomyces species revealed a core genome of 2018 genes, with 11,743 in the accessory genome, and 20,831 in the unique genome [50] while another one on 31 Streptomyces species revealed 2048 core genes, 9806 accessory and 17,840 unique genes [51]. Similarly, a comparative genomic analysis of 17 species of the genus Nocardiopsis revealed a core genome of 1993 genes and a pan genome of over 22,000 genes [52]. To identify and compare ortholog clusters, these studies used the pan genome analysis pipeline PGAP [53]. A second analysis using PGAP with 37 Amycoaltopsis genomes showed very similar results, albeit different exact numbers (Additional file 1: Figure S12). The core/pan-genome difference between both methods can be explained by leaving out $A$. nigrescens from the analysis and by the fact that the original NCBI annotations had to be used to prepare the input data for PGAP. Both analyses reveal a very small core genome compared to other studies. It is likely that this discrepancy results from the higher number of genomes compared in our study, which usually results in a lower core genome and shows the diversity of the genus.

The Amycolatopsis pan-genome is quite large and is still considered as "open". This shows that members of the genus have an extensive adaptive capacity. The COG analysis (Additional file 1: Figure S3) showed that a major part of the accessory and unique genes of the Amycolatopsis strains are involved in secondary metabolite biosynthesis and transport. Previous studies suggested that the diversity of secondary metabolites in bacteria is highly dependent on the bacterial genus [16, 38]. It is clear from this study that the capacity of members of the genus Amycolatopsis to produce diverse secondary metabolites is comparable to that of the genera Mycobacterium and Streptomyces [38].

When taking a closer look at the potential of Amycolatopsis strains to synthesize secondary metabolites different trends are apparent in the diversity and distribution of BGCs: I) Some BGCs were found in members of all four of the subgroups. These BGCs mainly encoded ectoines, non-NRPS derived siderophores, terpenes and RiPPs; no PKS or NPRS clusters fell into this grouping. These BGCs probably play a universal role in the metabolism of $A m y$ colatopsis, and therefore might be seen as core-secondary metabolite clusters. II) In contrast, a correlation with the subgroup phylogeny was shown for most of the common BGCs. These clusters have most likely been acquired through HGT in an ancestor strain, and have been retained throughout speciation. III) The extensive range of unique BGCs observed accounted for $67 \%$ of the diverse Amycolatopsis GCFs and seemed to be derived from recent HGT events. These clusters might be retained, if they enhance the ability of strains to colonize ecological niches, or might be lost, and/or replaced if no such advantage is realized [37].

Two previous studies on the diversity of secondary metabolites within actinobacterial taxa gave contradictory results on the relationship between phylogeny and diversity of BGCs. Doroghazi et al., found that in 860 actinobacterial genomes BGC diversity for PKS and NRPS genes correlated with phylogeny at the species level thereby revealing the importance of secondary metabolites for speciation [15]. In contrast, Cimermancic et al. reported that the highest BGC diversity was at the tips of phylogenetic trees, indicating that their diversification is phylogeny independent [16]. BGC diversity in the present study reflects both of these trends suggesting that vertical gene transfer might be the most important driver for the maintenance of common BGCs while recent HGT events independent of phylogeny, as seen as through the singletons and, phylogenetically independent cluster families might lead to further diversification. The tendency of phylogenetically related BGCs to be located at the same position in the genomes of Amycolatopsis supports the hypothesis that these BGCs may have arisen from the same ancestral strain. At the same time the observation that BGCs which belong to the same cluster family are present in distinctly related strains is in line with their distribution by HGT.

Previous studies on the diversity and evolution of Salinispora BGCs showed that a number of BGCs was fixed over globally distributed populations [54], though the highest diversity of Salinispora BGCs by far were derived from unique BGCs, on average 1-2 were found even within highly conserved species [14]. 
Similar observations to those outlined above can be made for Amycolatopsis where BGC diversity is derived mostly from singleton BGCs. As Amycolatopsis strains are not as closely related to one another as Salinispora strains, an average of 6-7 novel BGCs tend to be present in new species though BGC fixation beyond the species level was observed within the phylogenetic subgroups.

The majority of BGCs in Amycolatopsis genomes tend to be located in a region opposite the core region surrounding the origin of replication. This suggests that the acquisition of BGCs via HGT occurs preferentially in non-core regions of the genome. The distinction between core- and non-core-regions has previously been proposed for the genomes of $A$. mediterranei U32 [55], A. orientalis HCCB10007 [43] and A. methanolica 239 [41], where regions with a lower density of coding genes were observed and considered to be non-core-regions. In general, these regions correspond with the regions of high BGC diversity observed in the present study although the proposed variable regions are larger than the non-core-regions proposed for strains U32, HCCB10007 and 239. A similar phenomenon has been observed for Streptomyces where a core region in the linear chromosome around the replication origin is conserved, while the arms of the chromosome display a high variability and contain the majority of species specific sequences [56]. In this same study, it was also reported that the more phylogenetically distant the strains, the greater the size of the variable region. In the present study, it was found that within the closely related subgroups (groups A, B and D) the size of the hypervariable region opposite the $d n a A$ gene is smaller than in the distantly related subgroup (group C). All in all, our study is in agreement with the hypothesis that BGCs are located mainly in the non-core region, probably because insertions in essential gene clusters would in most cases prove to be lethal for the organism [56]. However, the fact that some BGCs, such as these coding for lantipeptides, are mainly located in the core region shows that BGC-location is not exclusively found in the hypervariable regions indicating that insertions in core regions are not necessary lethal.

In the present study it was not possible, as is the case of the more highly conserved genus Salinispora [57], to detect precise genomic islands, given the extreme genetic variation and small core genome though hypervariable regions were evident within the genetic subgroups. These hypervariable regions corresponded with the majority of BGCs, but showed no consistent structural similarities, as corresponding flanking regions, or conserved mobile elements. To establish whether a "pathway swapping" mechanism, as evident for Salinispora [14], is also true for Amycolatopsis, a larger number of more closely related strains needs to be analyzed.

\section{Conclusions}

A comparative analysis of the genus Amycolatopsis and its' biosynthetic potential revealed a highly variable gene content. All of the Amycolatopsis strains showed a small core-genome, but had a huge pan-genome indicating a great potential for the production of secondary metabolites. We were able to distinguish four phylogenetic sublineages within the genus Amycolatopsis, and four strains that formed distinct lineages in the phylogenetic tree. When comparing the phylogenetic resolution with the potential of Amycolatopsis strains to produce secondary metabolites an extensive diversity of BGCs was seen, most of which comes from clusters unique to the genus. Horizontal and vertical gene transfer seem equally important to drive and maintain the diversity of secondary metabolites. Among the vertically inherited clusters, a few extend across several phylogenetic lineages but most are specific for individual lineages. The observation that really novel clusters acquired through HGT were detected shows that related biosynthetic pathways can be transferred to unrelated strains through this mechanism. Further, it is evident that novel BGCs are mainly, but not exclusively incorporated into non-core hypervariable regions opposite the replication origin on the circular Amycolatopsis genomes.

\section{Methods}

\section{Amycolatopsis genomes}

All of the Amycolatopsis genome sequences available in December 2016 at the National Center for Biotechnology Information (NCBI) database [58] and the DOE Joint Genome Institute -Integrated Microbial Genomes \& Microbiomes (JGI-IMG) database [59], were used. Draft genomes that consisted of more than 300 contigs and sequences from single cell genomic approaches were omitted due to quality issues.

For the sequencing of the Amycolatopsis sp. H5 and KNN 50.9b genomes, sequencing libraries were prepared by applying Illumina TruSeq DNA PCR-Free Library Preparation Kits with a target insert size of 550 bp. Subsequent paired-end sequencing was performed on an Illumina HiSeq 1500 System (Illumina, San Diego, CA, USA) using HiSeq Reagent v3 Kits (Illumina, San Diego, CA, USA). Read length was $2 \times 250 \mathrm{bp}$. Base calling was performed with an in-house software platform [60]. To assemble the resultant reads, the gsAssembler software (Newbler) v2.8 was used. The genome sequence was submitted to the NCBI Prokaryotic Gene Annotation Pipeline for annotation.

\section{Comparative analysis of Amycolatopsis strains}

To elucidate the phylogenetic relationships between the Amycolatopsis strains a multilocus sequence typing approach based on the concatenation of seven housekeeping genes atpD, clpB, gap $A, \operatorname{gyr} B$, nuoD, pyrH and $r p o B$ 
was used. The single gene sequences were aligned using ClustalW, embedded in MEGA6.0 software [61], trimmed with respect to the reading frame and subsequently concatenated with the FaBox Fasta Alignment Joiner [62]. A maximum likelihood tree was generated using the Tamura-Nei Model with NNI (Nearest Neighbor Interchange) and 500 bootstrap replications was calculated with MEGA6.0 software.

Core-/pan-genome analysis was performed using the Bacterial Pan Genome Analysis (BPGA) tool [32]. To avoid bias derived from different annotations all of the genome sequences were newly annotated using PROKKA 1.2 with default settings [63]. As all six of the A. mediterranei genomes were highly similar A. mediterranei $\mathrm{S} 699$ was taken to represent the species to avoid bias. Orthologous genes were identified with the USEARCH algorithm [64] using a threshold of 0.5 . Variations of the similarity threshold to $0.3,0.4,0.6$ an 0.7 did not significantly alter the results, therefore the default threshold of 0.5 was chosen. Core-/pan-genome plots were calculated over 500 iterations. For comparative purposes an additional core-/pan-genome analysis was performed using the pan genome analysis pipeline PGAP [53]. Runs were performed using default settings under the MP and GF mode of PGAP.

To resolve the relationship of Amycolatopsis strains on the genus and species level the percentage of conserved proteins (POCP) was calculated as previously described [46], and the Average Nucleotide Identity based on the MUMmer algorithm (ANIm) was calculated with JSpecies using the default settings [65]. Graphical visualization of ANIm values was implemented with $\mathrm{R}$ version 3.3.3 [66].

\section{BGC and GCF identification}

The biosynthetic gene clusters of all of the Amycolatopsis strains were identified using antiSMASH 3.0 with default settings [10]. Identified clusters were compared using MultiGeneBlast [67]. Cluster boundaries were determined as previously described [34] and clusters were manually trimmed using Artemis [68].

Assigning gene clusters to GCFs was based on manual inspection of the antiSMASH output files, a comparison with multigeneblast and sequence comparison of KS and $\mathrm{C}$ domains was achieved using BLAST [69] and NaPDoS [70]. The following criteria had to be met for BGC clusters to be assigned to the same gene cluster family: I) The gene clusters had to have a similar architecture, II) The majority of genes included in the cluster needed to have the same function, but not necessarily in the same order. III) The majority of genes in the genome needed to have a BLAST similarity of at least $50 \%$ identity over an $80 \%$ coverage rate. IV) For modular PKS, NRPS and their hybrid clusters a BLAST similarity of the respective $\mathrm{KS}$ and $\mathrm{C}$ domains was considered. Hence, KS and C domains with the same modular position in the different clusters were compared. Clusters where the majority of $\mathrm{KS}$ and/or C domains shared a BLAST identity over $80 \%$ were considered to belong to the same GCF. Results were collected in a presence/absence matrix, with 1 representing the presence and 0 the absence of a GCF member in each of the Amycolatopsis strains. Hierarchical cluster analysis using the DICE coefficient with UPGMA (Unweighted Pair Group Method with Arithmetic mean) was performed with PAST [71]. Comparison of the Amycolatopsis phylogenetic tree with the BGC-dendrogram was performed with Dendroscope v3.5.7, using the Tanglegram algorithm [72].

For genetic networking, the Pfam-domains of each BGC were identified using HMMER 3.1b2 [73] with the respective Hidden Markov Models (HMM) obtained from the Pfam database [74]. A similarity index based on the absence or presence of Pfam domains was used to delineate BGC similarity, as previously described by Lin et al. [75] with the modifications of Cimermancic et al. [16]. A similarity threshold of 0.65 was chosen, because it best reflected the manually determined GCFs. The threshold was evaluated manually, as the threshold values of 0.5 [16] and 0.8 [76] described in previous publications were not found to be suitable to distinguish between the Amycolatopsis BGCs. The resulting similarity matrix was visualized with Cytoscape 3.4.0 [77].

Rarefaction curves displaying the relative BGC richness for each phylogenetic group were calculated from the BGC presence/absence matrix using EstimateS [78].

\section{BGC location}

To schematically display the relative positions of the common BGC clusters on the Amycolatopsis genomes, the approach previously described by Ziemert et al. [14] was used. First, the draft genomes were assembled as pseudocontigs on the phylogenetically closest complete genome as a reference using CONTIGuator v2.7 [79]. The circular genomes were linearized, using the $d n a A$ gene as the start for each linearized pseudocontig. If necessary, the reverse complement sequence was used for genome alignment. Second, the position of the respective BGCs on the complete genomes and on the pseudocontigs was annotated using geneious R9.1.6 [80]. Finally, the complete genomes and pseudocontigs were normalized in length to visually distinguish between the relative position of the BGCs on the genomes and pseudocontigs. The contigs were aligned to the closest related complete genome within the same phylogenetic group. The circular genomes were linearized and normalized in length. An overview of the of complete genome and pseudo contig synteny is shown in Additional file 1: Figure S13. 
To distinguish conserved regions from hypervariable regions on the Amycolatopsis genomes and pseudocontigs, and to identify genome rearrangements, the Harvest toolkit containing the Parsnp v1.2 tool for core genome alignment and Gingr 1.2 for visualization was used [81]. Due to the small core genome of Amycolatopsis, a core genome alignment for all of the strains was not feasible hence, core genome alignment for the phylogenetic subgroups that shared 85\% ANIm was performed. This excluded the genome sequences of $A$. halophila, A. marina, A. nigrescens, A. sacchari, A. taiwanensis and A. xylanica from this analysis.

BGC density plots were created with $\mathrm{R}$ version 3.3.3 [66]. Thereby, the genome was divided into 8 regions, and density plots were built showing the abundance of BGCs in each region, for each cluster type and for all cluster types in total.

\section{Additional files}

Additional file 1: Supplementary Figures. Figure S1-S13 (PDF $6871 \mathrm{~kb}$ )

Additional file 2: Table S1. Sequencing statistics for Amycolatopsis sp. $\mathrm{H} 5$ and Amycolatopsis sp. KNN50.9b. (DOCX $42 \mathrm{~kb}$ )

Additional file 3: Table S2. Basic features of Amycolatopsis genomes. (DOCX $110 \mathrm{~kb}$ )

Additional file 4: Table S3. POCP analysis. (XLSX $20 \mathrm{~kb}$ )

\section{Abbreviations}

AMS: Thermophilic Amycolatopsis methanolica subclade; ANI: Average Nucleotide Identity; AOS: Mesophilic/moderately thermophilic Amycolatopsis orientalis subclade; ATS: Thermophilic Amycolatopsis taiwanensis subclade; BGC: Biosynthetic gene cluster; GCF: Gene cluster family; HGT: Horizontal gene transfer; MIBiG: Minimum Information about a Biosynthetic Gene cluster; NRPS: Non-ribosomal peptide synthetase; PKS: Polyketide synthase; POCP: Percentage of Conserved Proteins; RiPP: Ribosomally synthesized and post-translationally modified peptides

\section{Acknowledgements}

The authors would like to thank Timo Niedermeyer for establishing the collaboration that secured the novel Amycolatopsis strains. Strains KNN 50.9b and $\mathrm{H} 5$ were isolated and characterized by Kanungnid Busarakam and Hamidah Idris of Newcastle University, UK. The authors are grateful to Evi Stegmann for valuable discussions.

\section{Funding}

This work was supported by the DZIF TTU 9.704. Alan T Bull and Michael Goodfellow are grateful to the Leverhulme Trust for the award of Emeritus Fellowships. The bioinformatics support of the BMBF funded project 'Bielefeld-Gießen Center for Microbial Bioinformatics - BiGi (Grant number 031A533)' within the German Network for Bioinformatics Infrastructure (de.NBI) is gratefully acknowledged. The funding bodies had no role in the design of the study, the preparation of the manuscript and the collection, analysis, and interpretation of data.

\section{Availability of data and materials}

Data from the following public databases was used for the analyses:

- Complete and draft genomes from the Joint Genome Institute (JGI) genome portal http://genome.jgi.doe.gov/

- Complete and draft genomes from the national (NCBI) assembly database https://www.ncbi.nlm.nih.gov/assembly/

- Reference biosynthetic gene clusters from the Minimum Information about a Biosynthetic Gene Cluster Database (MIBiG) http:// mibig.secondarymetabolites.org/
- This Whole Genome Shotgun project has been deposited at DDBJ/ ENA/GenBank under the accession NMUL00000000 (H5) and NMUK00000000 (KNN50.9b). The version described in this paper is version NMUL01000000 for Amycolatopsis sp. H5 and version NMUK01000000 for Amycolatopsis sp. KNN50.9b.

\section{Authors' contributions}

MAd carried out the comparative genomics analyses and wrote the paper. MAl wrote the bioinformatics scripts for the genomic analyses, and supported MAd with the comparative genomics analyses. HSA contributed the BGC density analysis and visualization with R. MG and ATB were responsible for overseeing the isolation and characterization of the novel Amycolatopsis sp. strains $\mathrm{H} 5$ and KNN50.9b. DW performed the POCP analysis. DW, AW and JK were responsible for sequencing the novel Amycolatopsis strains, and NZ guided the research and edited the manuscript. All authors read and approved the manuscript.

\section{Ethics approval and consent to participate}

Not applicable.

\section{Competing interests}

The authors declare that they have no competing interests.

\section{Publisher's Note}

Springer Nature remains neutral with regard to jurisdictional claims in published maps and institutional affiliations.

\section{Author details}

${ }^{1}$ Interfaculty Institute of Microbiology and Infection Medicine Tübingen, Microbiology/Biotechnology, University of Tübingen, Tübingen, Germany ${ }^{2}$ German Centre for Infection Research (DZIF), Partner Site Tübingen,

Tübingen, Germany. ${ }^{3}$ School of Biology, Newcastle University, Ridley Building 2, Newcastle upon Tyne NE1 7RU, UK. ${ }^{4}$ School of Biosciences, University of Kent, Canterbury CT2 7NJ, UK. ${ }^{5}$ Universität Bielefeld, Center for Biotechnology (CeBiTec), Bielefeld, Germany.

Received: 28 August 2017 Accepted: 21 May 2018

Published online: 01 June 2018

\section{References}

1. Katz L, Baltz RH. Natural product discovery: past, present, and future. J Ind Microbiol Biotechnol. 2016:43(2-3):155-76.

2. Newman DJ, Cragg GM. Natural products as sources of new drugs from 1981 to 2014. J Nat Prod. 2016;79(3):629-61.

3. Spellberg B. The future of antibiotics. Crit Care. 2014;18(3):228.

4. Medema MH, Fischbach MA. Computational approaches to natural product discovery. Nat Chem Biol. 2015;11(9):639-48.

5. Ziemert N, Alanjary M, Weber T. The evolution of genome mining in microbes - a review. Nat Prod Rep. 2016;33(8):988-1005.

6. Weissman KJ. The structural biology of biosynthetic megaenzymes. Nat Chem Biol. 2015;11(9):660-70.

7. Arnison PG, Bibb MJ, Bierbaum G, Bowers AA, Bugni TS, Bulaj G, Camarero JA, Campopiano DJ, Challis GL, Clardy J, et al. Ribosomally synthesized and post-translationally modified peptide natural products: overview and recommendations for a universal nomenclature. Nat Prod Rep. 2013;30(1):108-60.

8. Daum M, Herrmann S, Wilkinson B, Bechthold A. Genes and enzymes involved in bacterial isoprenoid biosynthesis. Curr Opin Chem Biol. 2009;13(2):180-8

9. Baltz RH. Gifted microbes for genome mining and natural product discovery. J Ind Microbiol Biotechnol. 2017:44(4-5):573-88.

10. Weber T, Blin K, Duddela S, Krug D, Kim HU, Bruccoleri R, Lee SY, Fischbach MA, Muller $\mathrm{R}$, Wohlleben W, et al. antiSMASH 3.0-a comprehensive resource for the genome mining of biosynthetic gene clusters. Nucleic Acids Res. 2015;43(W1):W237-43.

11. Traxler MF, Kolter R. Natural products in soil microbe interactions and evolution. Nat Prod Rep. 2015;32(7):956-70.

12. Davies J. Specialized microbial metabolites: functions and origins. J Antibiot (Tokyo). 2013;66(7):361-4.

13. Florez LV, Scherlach K, Gaube P, Ross C, Sitte E, Hermes C, Rodrigues A, Hertweck C, Kaltenpoth M. Antibiotic-producing symbionts dynamically transition between plant pathogenicity and insect-defensive mutualism. Nat Commun. 2017:8:15172. 
14. Ziemert N, Lechner A, Wietz M, Millan-Aguinaga N, Chavarria KL, Jensen PR. Diversity and evolution of secondary metabolism in the marine actinomycete genus Salinispora. Proc Natl Acad Sci U S A. 2014;111(12):E1130-9.

15. Doroghazi JR, Albright JC, Goering AW, Ju KS, Haines RR, Tchalukov KA, Labeda DP, Kelleher NL, Metcalf WW. A roadmap for natural product discovery based on large-scale genomics and metabolomics. Nat Chem Biol. 2014;10(11):963-8.

16. Cimermancic P, Medema MH, Claesen J, Kurita K, Wieland Brown LC, Mavrommatis K, Pati A, Godfrey PA, Koehrsen M, Clardy J, et al. Insights into secondary metabolism from a global analysis of prokaryotic biosynthetic gene clusters. Cell. 2014;158(2):412-21.

17. LPSN - List of procaryotic names with standing in nomenclature, Acessed May, 2017 [http://www.bacterio.net/amycolatopsis.html].

18. Bian J, Li Y, Wang J, Song FH, Liu M, Dai HQ, Ren B, Gao H, Hu X, Liu ZH, et al. Amycolatopsis marina sp. nov., an actinomycete isolated from an ocean sediment. Int J Syst Evol Microbiol. 2009;59(Pt 3):477-81.

19. Carlsohn MR, Groth I, Tan GY, Schutze B, Saluz HP, Munder T, Yang J, Wink J, Goodfellow M. Amycolatopsis saalfeldensis sp. nov., a novel actinomycete isolated from a medieval alum slate mine. Int J Syst Evol Microbiol. 2007;57(Pt 7):1640-6.

20. Lechevalier MP, Prauser H, Labeda DP, Ruan J-S. Two new genera of Nocardioform Actinomycetes: Amycolata gen. nov. and Amycolatopsis gen. nov. Int J Syst Evol Microbiol. 1986;36(1):29-37.

21. Majumdar S, Prabhagaran SR, Shivaji S, Lal R. Reclassification of Amycolatopsis orientalis DSM 43387 as Amycolatopsis benzoatilytica sp. nov. Int J Syst Evol Microbiol. 2006:56(Pt 1):199-204.

22. Tang SK, Wang Y, Guan TW, Lee JC, Kim CJ, Li WJ. Amycolatopsis halophila $s p$. nov., a halophilic actinomycete isolated from a salt lake. Int J Syst Evol Microbiol. 2010;60(Pt 5):1073-8.

23. Wink JM, Kroppenstedt RM, Ganguli BN, Nadkarni SR, Schumann P, Seibert $\mathrm{G}$, Stackebrandt $\mathrm{E}$. Three new antibiotic producing species of the genus Amycolatopsis, Amycolatopsis balhimycina sp. nov., A. tolypomycina sp. nov., A. vancoresmycina sp. nov., and description of Amycolatopsis keratiniphila subsp. keratiniphila subsp. nov. and A. keratiniphila subsp. nogabecina subsp. nov. Syst Appl Microbiol. 2003;26(1):38-46.

24. Labeda DP, Donahue JM, Williams NM, Sells SF, Henton MM. Amycolatopsis kentuckyensis sp. nov., Amycolatopsis lexingtonensis sp. nov. and Amycolatopsis pretoriensis sp. nov., isolated from equine placentas. Int J Syst Evol Microbiol. 2003:53(Pt 5):1601-5.

25. Huang Y, Pasciak M, Liu Z, Xie Q, Gamian A. Amycolatopsis palatopharyngis sp. nov., a potentially pathogenic actinomycete isolated from a human clinical source. Int J Syst Evol Microbiol. 2004;54(Pt 2):359-63.

26. Chen $\mathrm{S}, \mathrm{Wu} \mathrm{Q}$, Shen $\mathrm{Q}$, Wang $\mathrm{H}$. Progress in understanding the genetic information and biosynthetic pathways behind Amycolatopsis antibiotics, with implications for the continued discovery of novel drugs. Chembiochem. 2016;17(2):119-28.

27. He HY, Pan HX, Wu LF, Zhang BB, Chai HB, Liu W, Tang GL. Quartromicin biosynthesis: two alternative polyketide chains produced by one polyketide synthase assembly line. Chem Biol. 2012;19(10):1313-23.

28. Dobashi K, Matsuda N, Hamada M, Naganawa H, Takita T, Takeuchi T. Novel antifungal antibiotics octacosamicins a and B. I. Taxonomy, fermentation and isolation, physico-chemical properties and biological activities. J Antibiot (Tokyo). 1988;41(11):1525-32.

29. Lukezic T, Lesnik U, Podgorsek A, Horvat J, Polak T, Sala M, Jenko B, Raspor P, Herron PR, Hunter IS, et al. Identification of the chelocardin biosynthetic gene cluster from Amycolatopsis sulphurea: a platform for producing novel tetracycline antibiotics. Microbiology. 2013;159(Pt 12):2524-32.

30. Kunimoto S, Lu J, Esumi H, Yamazaki Y, Kinoshita N, Honma Y, Hamada M, Ohsono M, Ishizuka M, Takeuchi T. Kigamicins, novel antitumor antibiotics. I. Taxonomy, isolation, physico-chemical properties and biological activities. J Antibiot (Tokyo). 2003;56(12):1004-11.

31. Beemelmanns C, Ramadhar TR, Kim KH, Klassen JL, Cao S, Wyche TP, Hou Y, Poulsen M, Bugni TS, Currie CR, et al. Macrotermycins A-D, glycosylated macrolactams from a termite-associated Amycolatopsis sp. M39. Org Lett. 2017;19(5):1000-3

32. Chaudhari NM, Gupta VK, Dutta C. BPGA- an ultra-fast pan-genome analysis pipeline. Sci Rep. 2016;6:24373.

33. Zhang W, Du P, Zheng H, Yu W, Wan L, Chen C. Whole-genome sequence comparison as a method for improving bacterial species definition. J Gen Appl Microbiol. 2014;60(2):75-8.

34. Adamek M, Spohn M, Stegmann E, Ziemert N. Mining bacterial genomes for secondary metabolite gene clusters. Methods Mol Biol. 2017;1520:23-47.
35. Bentley SD, Chater KF, Cerdeno-Tarraga AM, Challis GL, Thomson NR, James KD, Harris DE, Quail MA, Kieser H, Harper D, et al. Complete genome sequence of the model actinomycete Streptomyces coelicolor A3(2). Nature. 2002:417(6885):141-7.

36. Ikeda H, Ishikawa J, Hanamoto A, Shinose M, Kikuchi H, Shiba T, Sakaki Y, Hattori M, Omura S. Complete genome sequence and comparative analysis of the industrial microorganism Streptomyces avermitilis. Nat Biotechnol. 2003;21(5):526-31.

37. Jensen PR. Natural products and the gene cluster revolution. Trends Microbiol. 2016;24(12):968-77.

38. Doroghazi JR, Metcalf WW. Comparative genomics of actinomycetes with a focus on natural product biosynthetic genes. BMC Genomics. 2013;14:611.

39. Komaki H, Ichikawa N, Oguchi A, Hamada M, Tamura T, Fujita N. Genome-based analysis of non-ribosomal peptide synthetase and type-l polyketide synthase gene clusters in all type strains of the genus Herbidospora. BMC Res Notes. 2015;8:548.

40. Huang JR, Ming H, Li S, Zhao ZL, Meng XL, Zhang JX, Tang Z, Li WJ, Nie GX. Amycolatopsis xuchangensis sp. nov. and Amycolatopsis jiguanensis sp. nov., isolated from soil. Antonie Van Leeuwenhoek. 2016;109(11):1423-31.

41. Tang $B$, Xie F, Zhao W, Wang J, Dai S, Zheng H, Ding X, Cen X, Liu H, Yu Y, et al. A systematic study of the whole genome sequence of Amycolatopsis methanolica strain 239T provides an insight into its physiological and taxonomic properties which correlate with its position in the genus. Synth Syst Biotechnol. 2016;1(3):169-86.

42. Goris J, Konstantinidis KT, Klappenbach JA, Coenye T, Vandamme P, Tiedje JM. DNA-DNA hybridization values and their relationship to whole-genome sequence similarities. Int J Syst Evol Microbiol. 2007;57(Pt 1):81-91.

43. Xu L, Huang H, Wei W, Zhong Y, Tang B, Yuan H, Zhu L, Huang W, Ge M, Yang $\mathrm{S}$, et al. Complete genome sequence and comparative genomic analyses of the vancomycin-producing Amycolatopsis orientalis. BMC Genomics. 2014;15:363.

44. Brigham RB, Pittenger RC. Streptomyces orientalis, n. $s p$, the source of vancomycin. Antibiot Chemother (Northfield). 1956;6(11):642-7.

45. Jeong H, Sim YM, Kim HJ, Lee YJ, Lee DW, Lim SK, Lee SJ. Genome sequences of Amycolatopsis orientalis subsp. orientalis strains DSM 43388 and DSM 46075. Genome Announc. 2013;1(4):e00545-13.

46. Qin $\mathrm{QL}$, Xie BB, Zhang XY, Chen XL, Zhou BC, Zhou J, Oren A, Zhang YZ. A proposed genus boundary for the prokaryotes based on genomic insights. J Bacteriol. 2014;196(12):2210-5.

47. Chun J, Oren A, Ventosa A, Christensen H, Arahal DR, da Costa MS, Rooney $A P, Y i \quad H, X u X W$, De Meyer $S$, et al. Proposed minimal standards for the use of genome data for the taxonomy of prokaryotes. Int J Syst Evol Microbiol. 2018;68(1):461-6.

48. Tan GY, Ward AC, Goodfellow M. Exploration of Amycolatopsis diversity in soil using genus-specific primers and novel selective media. Syst Appl Microbiol. 2006:29(7):557-69.

49. Fondi M, Karkman A, Tamminen MV, Bosi E, Virta M, Fani R, Alm E, Mclnerney JO. "every gene is everywhere but the environment selects": global Geolocalization of gene sharing in environmental samples through network analysis. Genome Biol Evol. 2016;8(5):1388-400.

50. Kim JN, Kim Y, Jeong Y, Roe JH, Kim BG, Cho BK. Comparative genomics reveals the Core and accessory genomes of Streptomyces species. J Microbiol Biotechnol. 2015;25(10):1599-605.

51. Tian X, Zhang Z, Yang T, Chen M, Li J, Chen F, Yang J, Li W, Zhang B, Zhang $Z$, et al. Comparative genomics analysis of Streptomyces species reveals their adaptation to the marine environment and their diversity at the genomic level. Front Microbiol. 2016;7:998.

52. Li HW, Zhi XY, Yao JC, Zhou Y, Tang SK, Klenk HP, Zhao J, Li WJ. Comparative genomic analysis of the genus Nocardiopsis provides new insights into its genetic mechanisms of environmental adaptability. PLoS One. 2013;8(4):e61528.

53. Zhao Y, Wu J, Yang J, Sun S, Xiao J, Yu J. PGAP: pan-genomes analysis pipeline. Bioinformatics. 2012;28(3):416-8.

54. Jensen PR, Williams PG, Oh DC, Zeigler L, Fenical W. Species-specific secondary metabolite production in marine actinomycetes of the genus Salinispora. Appl Environ Microbiol. 2007;73(4):1146-52.

55. Zhao W, Zhong Y, Yuan H, Wang J, Zheng H, Wang Y, Cen X, Xu F, Bai J, Han $X$, et al. Complete genome sequence of the rifamycin SV-producing Amycolatopsis mediterranei U32 revealed its genetic characteristics in phylogeny and metabolism. Cell Res. 2010;20(10):1096-108.

56. Choulet F, Aigle B, Gallois A, Mangenot S, Gerbaud C, Truong C, Francou FX, Fourrier C, Guerineau M, Decaris B, et al. Evolution of the terminal regions of the Streptomyces linear chromosome. Mol Biol Evol. 2006;23(12):2361-9. 
57. Penn K, Jenkins C, Nett M, Udwary DW, Gontang EA, McGlinchey RP, Foster B, Lapidus A, Podell S, Allen EE, et al. Genomic islands link secondary metabolism to functional adaptation in marine Actinobacteria. ISME J. 2009;3(10):1193-203.

58. Coordinators NR. Database resources of the National Center for biotechnology information. Nucleic Acids Res. 2017:45(D1):D12-7.

59. Markowitz VM, Chen IM, Palaniappan K, Chu K, Szeto E, Grechkin Y, Ratner A, Jacob B, Huang J, Williams P, et al. IMG: the integrated microbial genomes database and comparative analysis system. Nucleic Acids Res. 2012;40(Database issue):D115-22.

60. Wibberg D, Andersson L, Tzelepis G, Rupp O, Blom J, Jelonek L, Puhler A, Fogelqvist J, Varrelmann M, Schluter A, et al. Genome analysis of the sugar beet pathogen Rhizoctonia solani AG2-2lllB revealed high numbers in secreted proteins and cell wall degrading enzymes. BMC Genomics. 2016;17:245.

61. Tamura K, Stecher G, Peterson D, Filipski A, Kumar S. MEGA6: molecular evolutionary genetics analysis version 6.0. Mol Biol Evol. 2013;30(12):2725-9.

62. Villesen P. FaBox: an online toolbox for FASTA sequences. Mol Ecol Notes. 2007;7(6):965-8.

63. Seemann T. Prokka: rapid prokaryotic genome annotation. Bioinformatics. 2014;30(14):2068-9.

64. Edgar RC. Search and clustering orders of magnitude faster than BLAST. Bioinformatics. 2010;26(19):2460-1.

65. Richter M, Rossello-Mora R, Oliver Glockner F, Peplies J. JSpeciesWS: a web server for prokaryotic species circumscription based on pairwise genome comparison. Bioinformatics. 2016;32(6):929-31.

66. Development R. Core team: R: a language environment for statistical computing. In: R Foundation for Statistical Computing; 2008.

67. Medema MH, Takano E, Breitling R. Detecting sequence homology at the gene cluster level with MultiGeneBlast. Mol Biol Evol. 2013;30(5):1218-23.

68. Carver T, Berriman M, Tivey A, Patel C, Bohme U, Barrell BG, Parkhill J, Rajandream MA. Artemis and ACT: viewing, annotating and comparing sequences stored in a relational database. Bioinformatics. 2008:24(23):2672-6.

69. Altschul SF, Gish W, Miller W, Myers EW, Lipman DJ. Basic local alignment search tool. J Mol Biol. 1990;215(3):403-10.

70. Ziemert N, Podell S, Penn K, Badger JH, Allen E, Jensen PR. The natural product domain seeker NaPDoS: a phylogeny based bioinformatic tool to classify secondary metabolite gene diversity. PLoS One. 2012;7(3):e34064.

71. Hammer $\varnothing$, Harper DAT, Ryan PD. PAST: paleontological statistics software package for education. Palaeontol Electron. 2001;4(1):9pp.

72. Huson DH, Scornavacca C. Dendroscope 3: an interactive tool for rooted phylogenetic trees and networks. Syst Biol. 2012;61(6):1061-7.

73. HMMER 3.1b2 [http://hmmer.org/].

74. Finn RD, Coggill P, Eberhardt RY, Eddy SR, Mistry J, Mitchell AL, Potter SC, Punta M, Qureshi M, Sangrador-Vegas A, et al. The Pfam protein families database: towards a more sustainable future. Nucleic Acids Res. 2016:44(D1):D279-85.

75. Lin $\mathrm{K}$, Zhu L, Zhang DY. An initial strategy for comparing proteins at the domain architecture level. Bioinformatics. 2006;22(17):2081-6.

76. Schorn MA, Alanjary MM, Aguinaldo K, Korobeynikov A, Podell S, Patin N, Lincecum T, Jensen PR, Ziemert N, Moore BS. Sequencing rare marine actinomycete genomes reveals high density of unique natural product biosynthetic gene clusters. Microbiology. 2016:162(12):2075-86.

77. Su G, Morris JH, Demchak B, Bader GD. Biological network exploration with Cytoscape 3. Curr Protoc Bioinformatics. 2014;47:8. 13 11-24

78. Colwell RK, Elsensohn JE. EstimateS turns 20: statistical estimation of species richness and shared species from samples, with non-parametric extrapolation. Ecography. 2014;37(6):609-13.

79. Galardini M, Biondi EG, Bazzicalupo M, Mengoni A. CONTIGuator: a bacterial genomes finishing tool for structural insights on draft genomes. Source Code Biol Med. 2011;6:11.

80. Kearse M, Moir R, Wilson A, Stones-Havas S, Cheung M, Sturrock S, Buxton S, Cooper A, Markowitz S, Duran C, et al. Geneious basic: an integrated and extendable desktop software platform for the organization and analysis of sequence data. Bioinformatics. 2012;28(12):1647-9.

81. Treangen TJ, Ondov BD, Koren S, Phillippy AM. The harvest suite for rapid core-genome alignment and visualization of thousands of intraspecific microbial genomes. Genome Biol. 2014;15(11):524.

\section{Ready to submit your research? Choose BMC and benefit from:}

- fast, convenient online submission

- thorough peer review by experienced researchers in your field

- rapid publication on acceptance

- support for research data, including large and complex data types

- gold Open Access which fosters wider collaboration and increased citations

- maximum visibility for your research: over $100 \mathrm{M}$ website views per year

At BMC, research is always in progress.

Learn more biomedcentral.com/submissions 